The Astrophysical Journal, 634:426-435, 2005 November 20

(C) 2005. The American Astronomical Society. All rights reserved. Printed in U.S.A.

\title{
THE EFFECT OF STELLAR EVOLUTION ON SiC DUST GRAIN SIZES
}

\author{
Angela K. Speck and Grant D. Thompson \\ Department of Physics and Astronomy, University of Missouri, Columbia, MO 65211 \\ AND \\ ANNE M. HofmeisTeR \\ Department of Earth and Planetary Science, Washington University, St. Louis, MO 63130 \\ Received 2005 June 8; accepted 2005 August 4
}

\begin{abstract}
Stars on the asymptotic giant branch (AGB) produce dust in their circumstellar shells. The nature of the dustforming environment is influenced by the evolution of the stars, in terms of both chemistry and density, leading to an evolution in the nature of the dust that is produced. Carbon-rich AGB stars are known to produce silicon carbide ( $\mathrm{SiC}$ ). Furthermore, observations of the $\sim 11 \mu \mathrm{m} \mathrm{SiC}$ feature show that the spectral features change in a sequence that correlates with stellar evolution. We present new infrared spectra of amorphous $\mathrm{SiC}$ and show that the $\sim 9 \mu \mathrm{m}$ feature seen in both emission and absorption, and correlated with trends in the $\sim 11 \mu \mathrm{m}$ feature, may be due to either amorphous $\mathrm{SiC}$ or nanocrystalline diamond with a high proportion of $\mathrm{Si}$ substituting for $\mathrm{C}$. Furthermore, we identify $\mathrm{SiC}$ absorption in three ISO spectra of extreme carbon stars, in addition to the four presented by Speck and coworkers. An accurate description of the sequence in the IR spectra of carbon stars requires accounting for both $\mathrm{SiC}$ emission and absorption features. This level of detail is needed to infer the role of dust in the evolution of carbon stars. Previous attempts to find a sequence in the infrared spectra of carbon stars considered $\mathrm{SiC}$ emission features while neglecting $\mathrm{SiC}$ absorption features, leading to an interpretation of the sequence that inadequately describes the role of dust. We show that the evolutionary sequence in carbon star spectra is consistent with a grain size evolution such that dust grains get progressively smaller as the star evolves. The evolution of the grain sizes provides a natural explanation for the shift of the $\sim 11 \mu \mathrm{m} \mathrm{SiC}$ feature in emission and in absorption. Further evidence for this scenario is seen in both post-AGB star spectra and in meteoritic studies of presolar grains.
\end{abstract}

Subject headings: circumstellar matter — dust, extinction — infrared: stars — stars: carbon

\section{INTRODUCTION}

\subsection{Evolution during the Asymptotic Giant Branch Phase}

Stars in the mass range 1-8 $M_{\odot}$ eventually evolve up the asymptotic giant branch (AGB), where they begin to lose mass and form circumstellar shells of dust and gas. During their ascent of the AGB, mass-loss rates are expected to increase with the luminosity of the star, resulting in progressive optical thickening of circumstellar shells. Furthermore, the chemistry of these stars' atmospheres, and thus of their circumstellar shells, changes as a result of dredge-up of newly formed carbon from the Heburning shell. Due to the extreme ease of formation and stability of $\mathrm{CO}$ molecules, the chemistry in circumstellar shells is controlled by the $\mathrm{C} / \mathrm{O}$ ratio. If $\mathrm{C} / \mathrm{O}<1$, all the carbon is trapped in $\mathrm{CO}$, leaving oxygen to dominate the chemistry. Conversely, if $\mathrm{C} / \mathrm{O}>1$, all the oxygen is trapped in $\mathrm{CO}$, and carbon dominates the chemistry. Stars start their lives with cosmic $\mathrm{C} / \mathrm{O}$ ratios $(\approx 0.4)$ and are thus oxygen-rich. In some AGB stars, the dredge-up of newly formed carbon is efficient enough to raise $\mathrm{C} / \mathrm{O}$ above unity, and these stars are known as carbon stars. They are expected to have circumstellar shells dominated by amorphous or graphitic carbon grains, although other dust grains are also important (e.g., silicon carbide; $\mathrm{SiC}$ ).

As the mass-loss rate increases with the evolution of the AGB stars, the dust shells get thicker (both optically and geometrically), and these stars eventually become invisible at optical wavelengths and very bright in the infrared (IR). Such stars are known as "extreme carbon stars" (Volk et al. 1992, 2000). At this stage, intense mass loss depletes the remaining hydrogen in the star's outer envelope and terminates the AGB. Up to this point, the star has been making and dredging up carbon, and thus these shells have even more carbon available for dust production than optically bright (early) carbon stars (i.e., the $\mathrm{Si} / \mathrm{C}$ ratio decreases with the evolution of these stars). The rapid depletion of material from the outer envelope of the star means that this extremely high mass-loss phase must have a relatively short duration (a few $\times 10^{4} \mathrm{yr}$; Volk et al. 2000).

\subsection{Post-AGB Evolution}

Once the AGB star has exhausted its outer envelope, the AGB phase ends. During this phase, the mass loss virtually stops, and the circumstellar shell begins to drift away from the star. At the same time, the central star begins to shrink and heat up from $3000 \mathrm{~K}$ until it is hot enough to ionize the surrounding gas, at which point the object becomes a planetary nebula (PN). The short-lived post-AGB phase, as the star evolves toward to the PN phase, is also known as the proto-planetary nebula (PPN) phase. The detached dust shell drifts away from the central star, causing a PPN to have cool infrared colors from its cooling dust shell. Meanwhile, the optical depth of the dust shell decreases as it expands, allowing the central star to be seen and making such objects optically bright. The effect of decreasing optical depth and cooling dust temperatures changes the spectrum of the circumstellar envelope, revealing features that were hidden during the AGB phase.

\subsection{Observing Dust around Carbon Stars}

Circumstellar shells of carbon stars are expected to be dominated by amorphous or graphitic carbon grains. These dust species do not have diagnostic infrared features and merely contribute 
to the dust continuum emission. However, silicon carbide ( $\mathrm{SiC}$ ) does exhibit a strong infrared feature, which can be exploited when studying carbon stars.

In the context of stardust, $\mathrm{SiC}$ has been of great interest since its formation was predicted by condensation models for carbonrich circumstellar regions (e.g., Friedemann 1969; Gilman 1969). Gilra $(1971,1972)$ predicted that $\mathrm{SiC}$ should reemit absorbed radiation as a feature in the $10-13 \mu \mathrm{m}$ region. Following these predictions, a broad infrared (IR) emission feature at $\sim 11.4 \mu \mathrm{m}$ that is observed in the spectra of many carbon stars has been attributed to solid SiC particles (e.g., Hackwell 1972; Treffers \& Cohen 1974). Indeed, $\mathrm{SiC}$ is now believed to be a significant constituent of the dust around carbon stars. Although Goebel et al. (1995) and Clément et al. (2003) adopted the name 11+ $\mu \mathrm{m}$ feature to reflect the variation in observed peak positions in astronomical spectra, this implies that the features always occur longward of $11 \mu \mathrm{m}$. We have adopted $\sim 11 \mu \mathrm{m}$ to reflect the variations in peak positions of this feature.

The effect of the evolution of the density of the dust shell on observed features, and particularly on the $\sim 11 \mu \mathrm{m}$ feature, has been discussed extensively (e.g., Cohen 1984; Baron et al. 1987; Willems 1988; Chan \& Kwok 1990; Goebel et al. 1995; Speck et al. 1997; Sloan et al. 1998; see $\S 3.1$ ). Not all these previous works agree. All studies concur that the increasing optical depth leads to decreasing color temperatures from the stars as the stellar photosphere becomes hidden from view and the dust from which we receive light becomes progressively cooler. At the same time, the $\sim 11 \mu \mathrm{m}$ feature tends to become weaker (relative to the continuum) and more flatly topped (less sharply peaked), and possibly broader. Various explanations of this behavior have been proposed, but none are entirely satisfactory. Initially, the diminution of the $\sim 11 \mu \mathrm{m}$ feature was attributed to coating of the grains by amorphous carbon (e.g., Baron et al. 1987; Chan \& Kwok 1990). However, meteoritic data do not support this hypothesis (see $\S 1.4$ ). Moreover, more recent studies of the $\sim 11 \mu \mathrm{m}$ feature have shown that it is consistent with $\mathrm{SiC}$ self-absorption, i.e., absorption by cooler $\mathrm{SiC}$ particles located in the outer part of the dust shell, where they can absorb the $\mathrm{SiC}$ emission feature produced by warmer $\mathrm{SiC}$ closer to the central star (Speck et al. 1997). Indeed, Speck et al. (1997) showed that every star in their sample whose underlying continuum temperature satisfied $T_{\text {col }}<1200 \mathrm{~K}$ was best fitted by self-absorbed $\mathrm{SiC}$, whether the $\sim 11 \mu \mathrm{m}$ feature was in net emission or net absorption. As the dust shell reaches extreme optical depths, the $\sim 11 \mu \mathrm{m}$ feature will eventually be seen in net absorption. However, these absorption features are rare and have mostly been ignored in discussions of evolutionary sequences in carbon star spectra.

\subsection{Meteoritic Evidence}

Silicon carbide is an important presolar grain found in meteorites. The isotopic compositions of presolar grains indicate that they originated outside the solar system. Presolar SiC was first discovered by Bernatowicz et al. (1987) and since then has been the focus of a great deal of laboratory work (see Bernatowicz et al. 2005 and references therein). The most important findings of this work are (1) that most of the $\mathrm{SiC}$ presolar grains were formed around carbon stars; $(2)$ that nearly all $(\sim 90 \%)$ are of the cubic $\beta$ polytype ${ }^{1}(3)$ that with one exception, $\mathrm{SiC}$ grains are never found

\footnotetext{
1 Silicon carbide can form into numerous different crystal structures, known as polytypes. $\beta$-SiC is the cubic polytype and is now believed to be the dominant form of SiC forming around carbon stars; see $\S 1.4$, and Speck et al. $(1997,1999)$ and Daulton et al. (2003).
}

in the cores of carbon presolar grains (unlike other carbides$\mathrm{TiC}, \mathrm{ZrC}$, and $\mathrm{MoC}$ ); and (4) that the grain size distribution is large $(1.5 \mathrm{~nm}-26 \mu \mathrm{m})$, with most grains in the $0.1-1 \mu \mathrm{m}$ range but with single-crystal grains that can exceed $20 \mu \mathrm{m}$ in size.

Observations of the $\sim 11 \mu \mathrm{m}$ feature have been compared with laboratory spectra of various forms of $\mathrm{SiC}$, and after some false starts it has now been attributed to $\beta$-SiC, matching the information retrieved from meteoritic samples (Speck et al. 1999; Clément et al. 2003). However, there are still some discrepancies between observational and meteoritic evidence (most notably related to grain size). Studies of meteoritic $\mathrm{SiC}$ grains can aid our understanding of their evolution.

\subsection{Purpose}

The goal of this paper is to extract the evolution of the dust from the infrared spectra of carbon-rich AGB stars (both optically bright and "extreme") and post-AGB stars. In $\S 2$ we present new infrared spectra of amorphous silicon carbide that may explain some of the features seen in the carbon star spectral sequences. In $\S 3$ we review studies of the infrared spectra of carbon stars that demonstrate the changes in spectral features associated with stellar evolution. We also present new detections of $\sim 11 \mu \mathrm{m}$ absorption features in extreme carbon stars, which are essential to our understanding of the dust grain evolution. In $\S 4$ we present a dust evolution sequence to explain the spectral changes in carbon stars. The summary and conclusions are in $\S 5$.

\section{NEW SPECTRA OF AMORPHOUS SILICON CARBIDE}

\subsection{Experimental Samples and Techniques}

Superior Graphite donated the bulk $\beta$-SiC. The purity is $99.8 \%$. Bulk $\alpha$-SiC (purity $=99.8 \%$ ) in the $6 \mathrm{H}$ polytype was purchased from Alfa/Aesar (Lot c19h06). The grain size varied from 1 to $25 \mu \mathrm{m}$. Nanocrystals of $2-5 \mathrm{~nm}$ size produced by gasphase combustion (Axelbaum et al. 1996) best match the X-ray diffraction pattern of $\beta$-SiC but contain some $6 \mathrm{H}$ as well (for details see Hofmeister et al. 2000). This sample may also contain $\mathrm{SiC}$ in the diamond structure, as synthesized by Kimura \& Kaito (2003) and discussed further below. We designate this sample as "nano- $\beta+\alpha$." These three samples were studied by Speck et al. (1999) and Speck \& Hofmeister (2004). Nanocrystals were also purchased from Nanostructured and Amorphous Materials, Inc. Amorphous $\mathrm{SiC}$ of $97.5 \%$ purity consists of $10 \times 100 \mathrm{~nm}^{2}$ laths (stock $4630 \mathrm{js}$ ). Nano- $\beta$ of $97 \%$ purity consists of $20 \mathrm{~nm}$ particles (stock 4640ke). Diamond of $95 \%$ purity consists of $3 \mathrm{~nm}$ particles (stock 1302jgy). Most SiC samples were dark gray, indicative of excess $\mathrm{C}$ or $\mathrm{Si}$, e.g., inclusions of these elements. Nanodiamond is light gray, presumably due to graphite impurities. The $\alpha-\mathrm{SiC}$ is pale gray to amber and has little in the way of impurities.

Mid-IR spectra were obtained from $\sim 450$ to $4000 \mathrm{~cm}^{-1}(2.2$ to $25 \mu \mathrm{m}$ ) at $2 \mathrm{~cm}^{-1}$ resolution using a liquid-nitrogen-cooled $\mathrm{HgCdTe}$ detector, a $\mathrm{KBr}$ beam splitter, and an evacuated Bomem DA 3.02 Fourier transform interferometer. Thin films were created through compression in a diamond anvil cell (DAC), which was used as a sample holder and for the reference spectrum. Efforts were made to cover the entire diamond tip $(0.6 \mathrm{~mm}$ diameter) with an even layer of sample, but slight irregularities in the thickness were inevitable. Far-IR data were obtained from powder dispersed in petroleum jelly on a polyethylene card from $\sim 150$ to $650 \mathrm{~cm}^{-1}(\sim 15$ to $67 \mu \mathrm{m})$ using a DTGS ${ }^{2}$ detector and mylar beam splitter. A spectrum of nanodiamond (which

\footnotetext{
${ }^{2}$ Deuterated triglycine sulfate.
} 


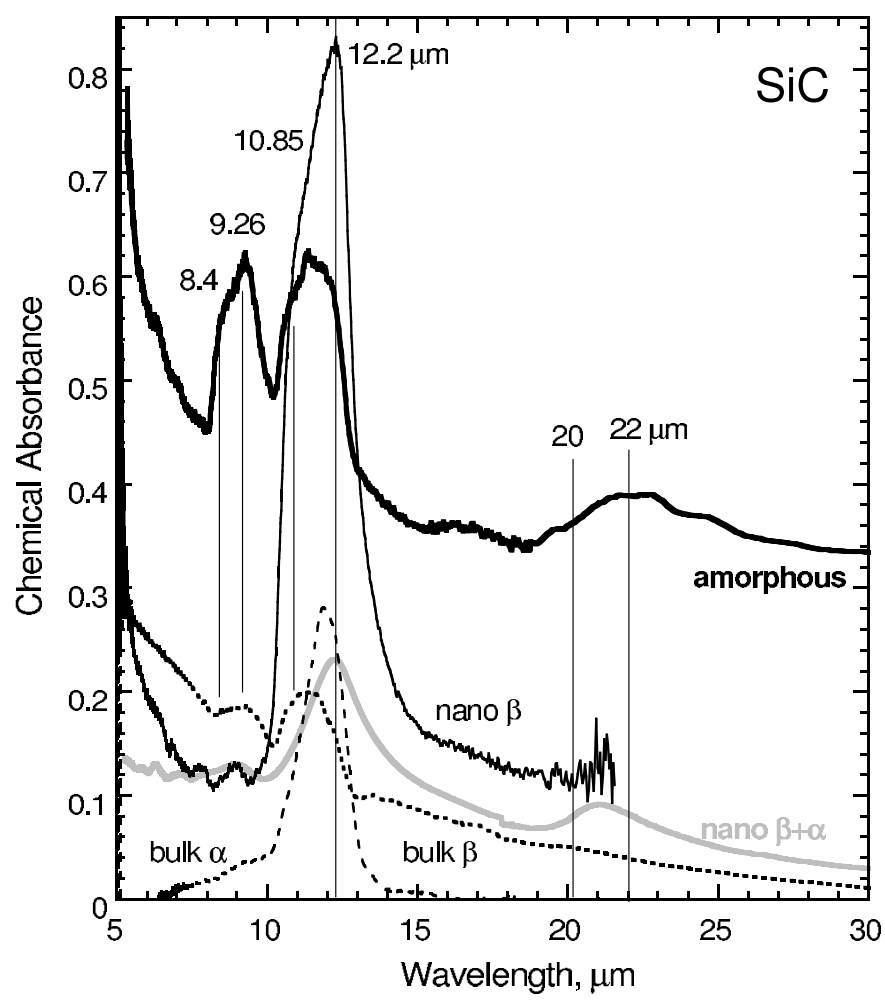

FIG. 1.-Merged far- and mid-IR spectra of various samples of SiC. Heavy solid line: Amorphous SiC. Light solid line: Nano- $\beta$. Gray solid line: Nano- $\beta+\alpha$. Dotted line: Bulk $\beta$. Dashed line: Bulk $\alpha$. Mid-IR segments $(\lambda=5-25 \mu \mathrm{m})$ are raw data. Far-IR data were scaled to match, but the merging and scaling are equivocal.

should be featureless) was subtracted to remove the effect of scattering. For the bulk and nano- $\beta+\alpha$ sample, a thin film was made in a DAC, and a helium bolometer served as the detector. Far- and mid-IR spectra were scaled to match in the region of overlap and merged. For procedural details, see Speck et al. (1999) or Hofmeister et al. (2003).

\subsection{Laboratory Results}

The two nanocrystalline samples have spectra that closely resemble each other, whereas the spectrum of the amorphous sample is most similar to that of bulk $\beta$-SiC (Fig. 1). All samples have three peak complexes centered near 9,11 , and $21 \mu \mathrm{m}$. The number and positions of the peaks constituting each com-

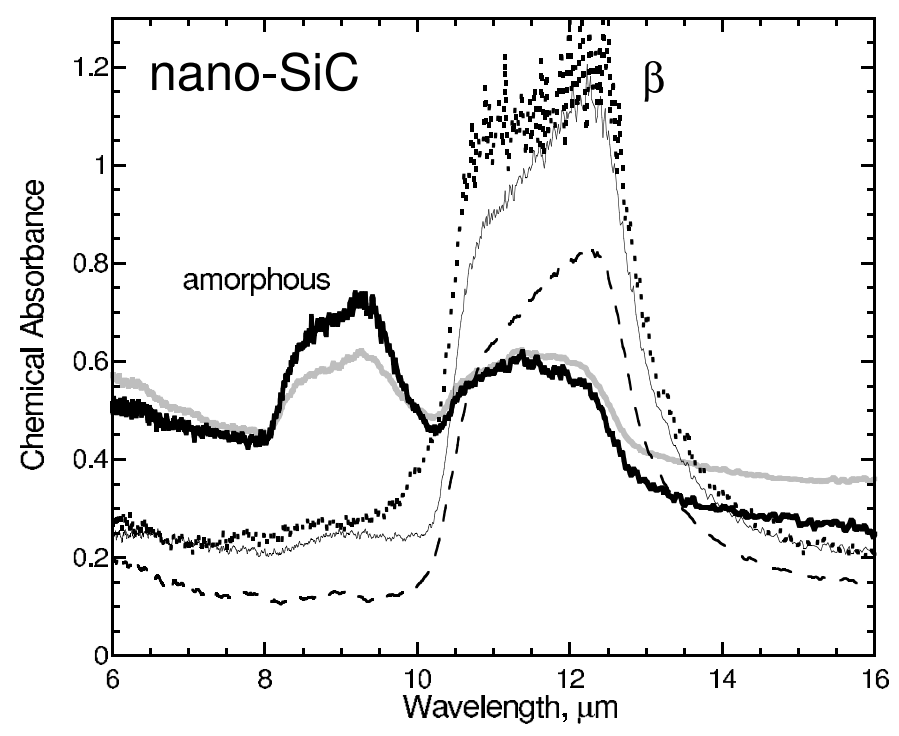

FIG. 2.-Dependence of spectra on thickness. Light lines, Nano- $\beta$; heavy lines, amorphous SiC. Dashed line, Thinest nano- $\beta$ sample; dotted line, thickest nano- $\beta$ sample; thick black line, thinnest amorphous sample; thick gray line, thickest amorphous sample. Raw data are shown.

plex vary, and the relative intensities of the complexes vary among the four samples (Table 1). Bulk $\alpha$-SiC shows only the $11 \mu \mathrm{m}$ complex. The shoulders in the spectrum shown are due to interference fringes, as the overtone combination peaks in this area are much weaker (e.g., Hofmeister et al. 2000). As it is clear from Figure 1 that the nanosamples have spectra unlike that of $\alpha-\mathrm{SiC}$ (and $\alpha$-SiC is absent from presolar grains; see $\S 1.4$ ), the remainder of the discussion concerns the amorphous and $\beta$-SiC samples.

Some of the spectral differences are due to grain size. From Figure 2 (and Fig. 2 in Hofmeister et al. 2000), as the sample thickens, the LO (longitudinal optic) shoulder increases in intensity relative to the main peak ( $\mathrm{TO}$, transverse optic). This occurs because of light leakage (see Hofmeister et al. 2003 for a detailed explanation). The nanocrystalline samples produce spectra close to the expected intrinsic profile (see Spitzer et al. 1959), because their very fine grain sizes aid production of a thin, uniform film that covers the diamond tip. The rounded profiles for $\beta$ and the amorphous $\mathrm{SiC}$ are attributed to production of less perfect films from these much larger particle sizes. The difference in relative intensity of the 11 and $9 \mu \mathrm{m}$ peaks between the

TABLE 1

Spectral Parameters of SiC in Various Forms

\begin{tabular}{|c|c|c|c|c|c|}
\hline Parameter & Bulk $\beta$ & Amorphous & Nano- $\beta$ & Nano- $\beta+\alpha$ & Bulk $\alpha$ \\
\hline Grain size $(\mathrm{nm})$ & $>1000$ & $10-100$ & 3 & $2-5$ & $\ldots$ \\
\hline Shoulder $(\mathrm{LO} ; \mu \mathrm{m}) \ldots \ldots \ldots \ldots \ldots \ldots \ldots \ldots$ & 8.4 & 8.4 & $\ldots$ & $\ldots$ & 7.66 (sharp) \\
\hline 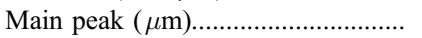 & 9.3 & 9.26 & 8.95 & 9.0 & $\ldots$ \\
\hline Shoulder $(\mathrm{LO} ; \mu \mathrm{m}) \ldots \ldots \ldots \ldots \ldots \ldots \ldots \ldots$ & 10.58 & 10.6 & 10.85 & $\ldots$ & 10.55 \\
\hline Barycenter or main $(\mu \mathrm{m}) \ldots \ldots \ldots \ldots \ldots . . . . . .$. & 11.30 & 11.43 & $\ldots$ & 12.14 & 11.85 \\
\hline Main peak or shoulder $(\mu \mathrm{m}) \ldots \ldots \ldots \ldots$ & 12.1 & 12.2 & 12.27 & 12.14 & 12.23 \\
\hline Peak $(\mu \mathrm{m})$ & $\ldots$ & 16.3 & $\ldots$ & $\ldots$ & 16.02 (sharp) \\
\hline Shoulder $(\mu \mathrm{m})$ & $\ldots$ & 20 & $\ldots$ & $\ldots$ & $\ldots$ \\
\hline 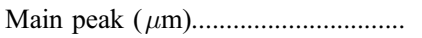 & 20.5 & 22 & $\ldots$ & 21 & $\ldots$ \\
\hline 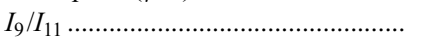 & $\sim 0.35$ & 0.73 & 0.050 & 0.15 & $\cdots$ \\
\hline 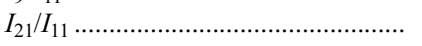 & $\sim 0.1$ & $\sim 0.26$ & $\ldots$ & $\sim 0.3^{\mathrm{a}}$ & $\cdots$ \\
\hline
\end{tabular}

NoTE.-Peak positions obtained from thinnest samples examined.

${ }^{a}$ Intensity is very uncertain, as the $\sim 21 \mu \mathrm{m}$ peak was measured using a dispersion. 
two spectra acquired for amorphous $\mathrm{SiC}$ may be due to different film thicknesses and amounts of light leakage as well. However, the relative intensity of this pair varied among the spectra of the $\beta$-SiC sample but was consistent for nano- $\beta+\alpha$ (Fig. 2 from Hofmeister et al. 2000), suggesting an impurity as the origin.

As observed by Speck \& Hofmeister (2004), the relative intensity of the 21 and $11 \mu \mathrm{m}$ peaks varied among the samples studied. This observation is supported by the two additional samples studied here, and intensities of the 21 and $9 \mu \mathrm{m}$ peaks are roughly correlated (Table 1). A feature similar to the $9 \mu \mathrm{m}$ peak has been observed in nanocrystals with varying proportions of $\mathrm{Si}$ and $\mathrm{C}$ by Kimura and colleagues. Carbon films that contain about $30 \%$ Si made by evaporation have a peak at $9.5 \mu \mathrm{m}$ (Kimura \& Kaito 2003). From electron diffraction, the structure of the films is nanodiamond, and small amounts of $\beta$-SiC are also present (Kimura \& Kaito 2003). Pure diamond structure should not have an IR peak, but impurity bands are well known, such as those due to nitrogen. Apparently, the Si-C stretch in the diamond structure differs from that in the derivative structure of $\beta$-SiC. Higher frequency is consistent with the lattice constant being smaller for diamond than for SiC.

Ion-sputtered carbon films with proportions of $10 \%, 30 \%$, and $50 \%$ Si similarly contain particles of nanodiamond and IR absorption bands at 9.5 and $21 \mu \mathrm{m}$. The $30 \%$ film has the most intense $9.5 \mu \mathrm{m}$ peak, whereas the $50 \%$ Si film contains nanocrystals of $\beta$-SiC in addition to the solid-solution nanodiamonds and weak peaks at 11.3 and $12.3 \mu \mathrm{m}$ (Kimura et al. 2005b).

Nanoparticles produced by radio-frequency plasma (Kimura et al. 2005a) absorb at 8.2 (shoulder), 9.2, a doublet at 11.2 and 11.7 , and a moderately intense band at $21 \mu \mathrm{m}$. The structure is nanodiamond. $\beta$-SiC peaks were rarely seen in the electron diffraction results, suggesting that the IR bands near $11 \mu \mathrm{m}$ result from short-range order (Kimura et al. 2005a). These authors attribute the $21 \mu \mathrm{m}$ band to excess carbon that may be present in interstitial sites in the solid-solution nanodiamond particles. The present data corroborate and augment their observations. Bulk $\beta$-SiC, nanocrystalline material, and amorphous nanosamples all behave similarly. In the amorphous sample the structural control is lost, so that the sample has places where the $\beta$-SiC structure occurs over the nearest neighbors and places where the nanodiamond structure occurs. These are approximately in equal proportions. Then there are local sites of excess $\mathrm{C}$ giving the $21 \mu \mathrm{m}$ band. That the 21 and $9 \mu \mathrm{m}$ peaks are strongest in the amorphous material corroborates assignment of these peaks to excess $\mathrm{C}$ in $\mathrm{SiC}$, one of the possibilities proposed by Speck \& Hofmeister (2004).

Therefore, the $9 \mu \mathrm{m}$ peak is indicative of Si-C locally in a diamond structure, and the $11 \mu \mathrm{m}$, of $\mathrm{Si}-\mathrm{C}$ locally in an $\mathrm{SiC}$ polytype. These peaks are found in amorphous $\mathrm{SiC}$ (observed here) and nanodiamond crystals (Kimura \& Kaito 2003; Kimura et al. 2005a, 2005b). The ratios of these peaks are not correlated, but depend on the $\mathrm{Si} / \mathrm{C}$ ratio of the material. Either amorphous $\mathrm{SiC}$ or nanocrystalline diamond with a high proportion of $\mathrm{Si}$ substituting for $\mathrm{C}$ is a good candidate for the carrier of the $9 \mu \mathrm{m}$ (see $\S 3.1)$ and $21 \mu \mathrm{m}(\S 3.3)$ features.

\subsection{Comparison with Astronomical Environments}

Figure 3 compares the amorphous $\mathrm{SiC}$ spectrum with the observed double-peaked emission feature seen in the spectrum of Y CVn (original published in Speck et al. 1997). Amorphous SiC provides peaks at the same positions as those observed in the double-peaked feature, but not in the same intensity ratio.

The shorter wavelength peak is due to excess carbon forming diamond-like structures in the amorphous or nanocrystalline

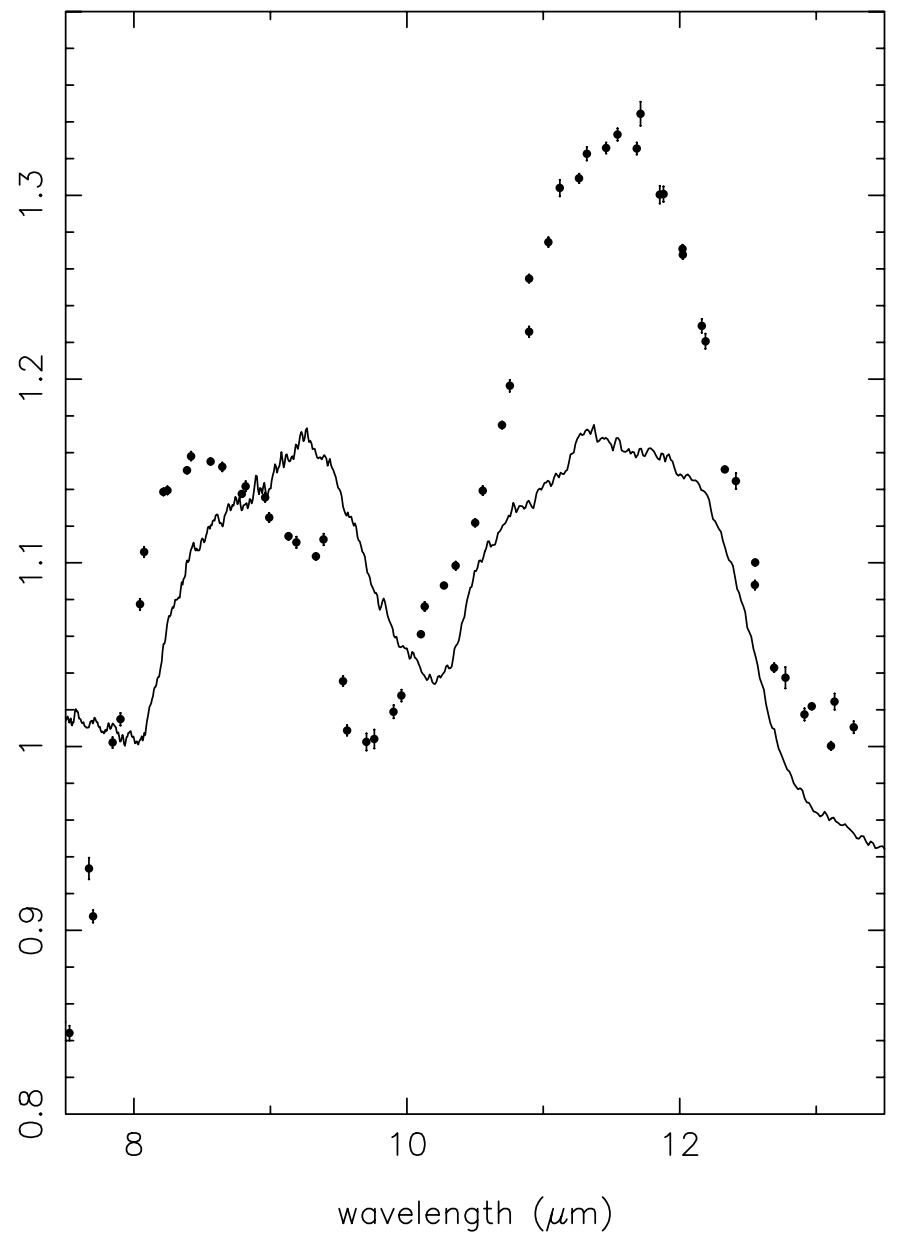

FIG. 3.-Comparison of double-peaked observed astronomical feature in the continuum-divided spectrum of Y CVn ( filled circles) with laboratory spectrum of amorphous $\mathrm{SiC}$ (solid line).

$\mathrm{SiC}$ grains. The relative strengths of the two peaks in the laboratory spectra are controlled by the concentration of excess carbon in the grains. Therefore, we can manipulate the composition of the grains to obtain bigger or smaller short-wavelength peaks, relative to the longer wavelength $\mathrm{Si}-\mathrm{C}$ peak. Furthermore, there may be a contribution to the $\mathrm{Si}-\mathrm{C}$ part of the spectrum from the $\beta$-SiC also present in the circumstellar shells, further enhancing the longer wavelength peak relative to the shorter one. Figure 4 shows the effect of mixing amorphous and crystalline $\mathrm{SiC}$ to varying degrees.

Comparing Figures 4 and 5 shows that the double-peaked amorphous $\mathrm{SiC}$ spectrum in combination with $\beta$-SiC can explain the variety of absorption features observed in the spectra of extreme carbon stars (see also $\S 3.2$ ). The differences in peak positions between the laboratory spectra and the observed astronomical absorption features are due to the effect of self-absorption and grain size, which is discussed in $\S 4$.

\section{SPECTROSCOPIC STUDIES OF CARBON STARS}

\subsection{IRAS LRS Studies of Carbon Stars}

The spectra of carbon stars change with the evolution of the star. Several studies exist of the evolution of the $\sim 11 \mu$ m feature in carbon star spectra based on Infrared Astronomical Satellite (IRAS) Low Resolution Spectrometer (LRS) data, but they are somewhat contradictory. The majority of carbon stars exhibit the $\sim 11 \mu \mathrm{m}$ feature in emission. With the exception of Speck 


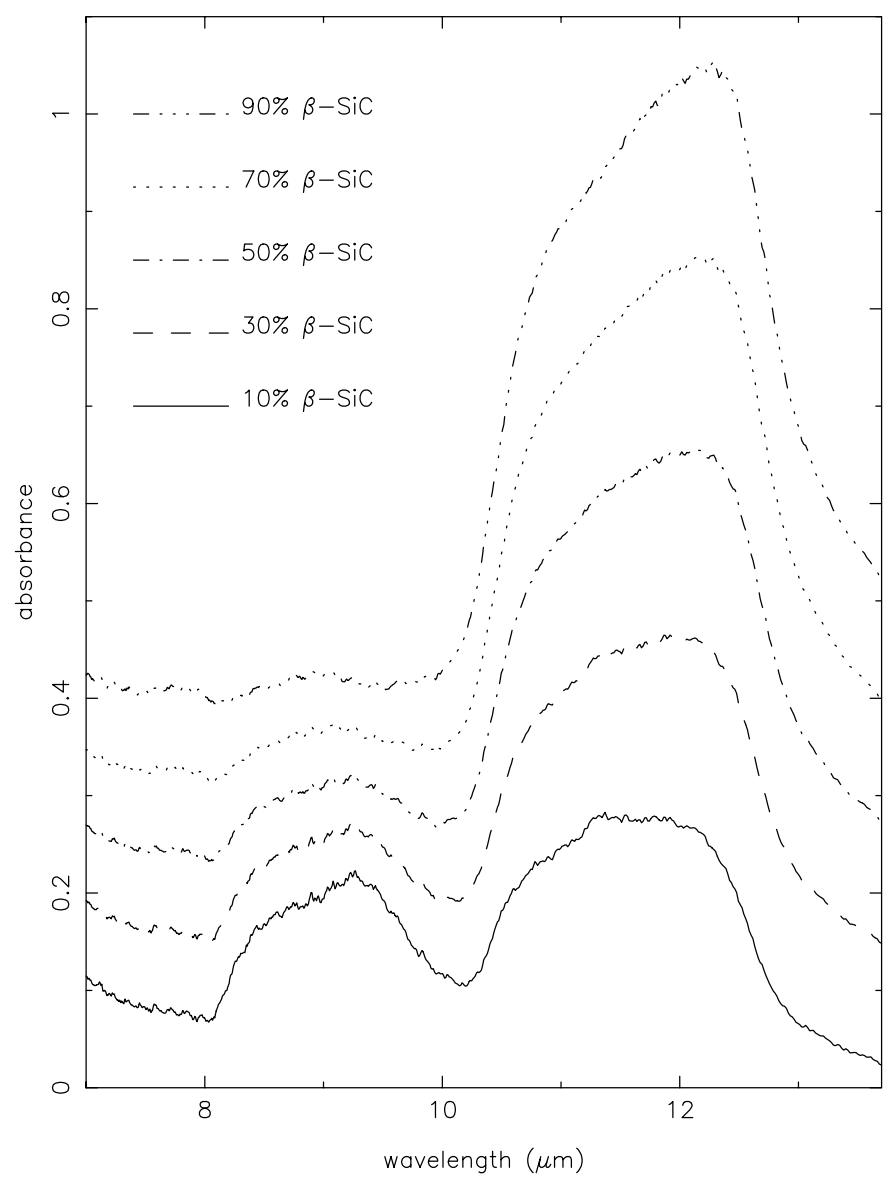

FIG. 4.-Effect of mixing amorphous and $\beta$-SiC.

et al. (1997), all attempts to understand the sequence of spectral features fail to include the $\mathrm{SiC}$ absorption feature. Here is the summary of the accepted evolutionary trends: Early in the carbon star phase, when the mass-loss rate is low and the shell is optically thin, the $\sim 11 \mu \mathrm{m} \mathrm{SiC} \mathrm{emission} \mathrm{feature} \mathrm{is} \mathrm{strong,} \mathrm{nar-}$ row, and sharp. As the mass loss increases and the shell becomes optically thicker, the $\mathrm{SiC}$ emission feature broadens, flattens, and weakens. Finally, once the mass-loss rate is extremely high and the shell is extremely optically thick, the $\mathrm{SiC}$ feature appears in absorption. Figure 6 shows spectra from Speck et al. (1997) that demonstrate these trends. Once the AGB phase ends, the dust thins and cools and we begin to see new features that are indicative of the dust in the extreme carbon star phase.

Little-Marenin (1986) found that the majority of $\sim 11 \mu \mathrm{m}$ emission features peak at $11.15 \mu \mathrm{m}$, with only $4 \%$ peaking longward of $11.6 \mu \mathrm{m}$. Baron et al. (1987) found that as the continuum temperature decreases and the peak continuum strength of the $\sim 11 \mu \mathrm{m}$ emission feature diminishes, the peak position tends to move to longer wavelengths (from $\sim 11.3$ to $\sim 11.7 \mu \mathrm{m}$ ). This trend is demonstrated in Figure 6. Willems (1988) and Goebel et al. (1995) found that the stars with high continuum temperatures tended to have the $\sim 11 \mu \mathrm{m}$ emission feature at $11.7 \mu \mathrm{m}$, with the $11.3 \mu \mathrm{m}$ feature arising in the spectra of stars with cool continuum temperatures, an obvious contradiction to the work of Baron et al. (1987). Speck et al. (1997) used a much smaller sample of ground-based United Kingdom Infrared Telescope (UKIRT) CGS3 spectra and found no correlation between the peak position of the $\sim 11 \mu \mathrm{m}$ feature and the continuum temperature.

Baron et al. (1987) and Goebel et al. (1995) noticed that the decreasing continuum temperature is accompanied by the

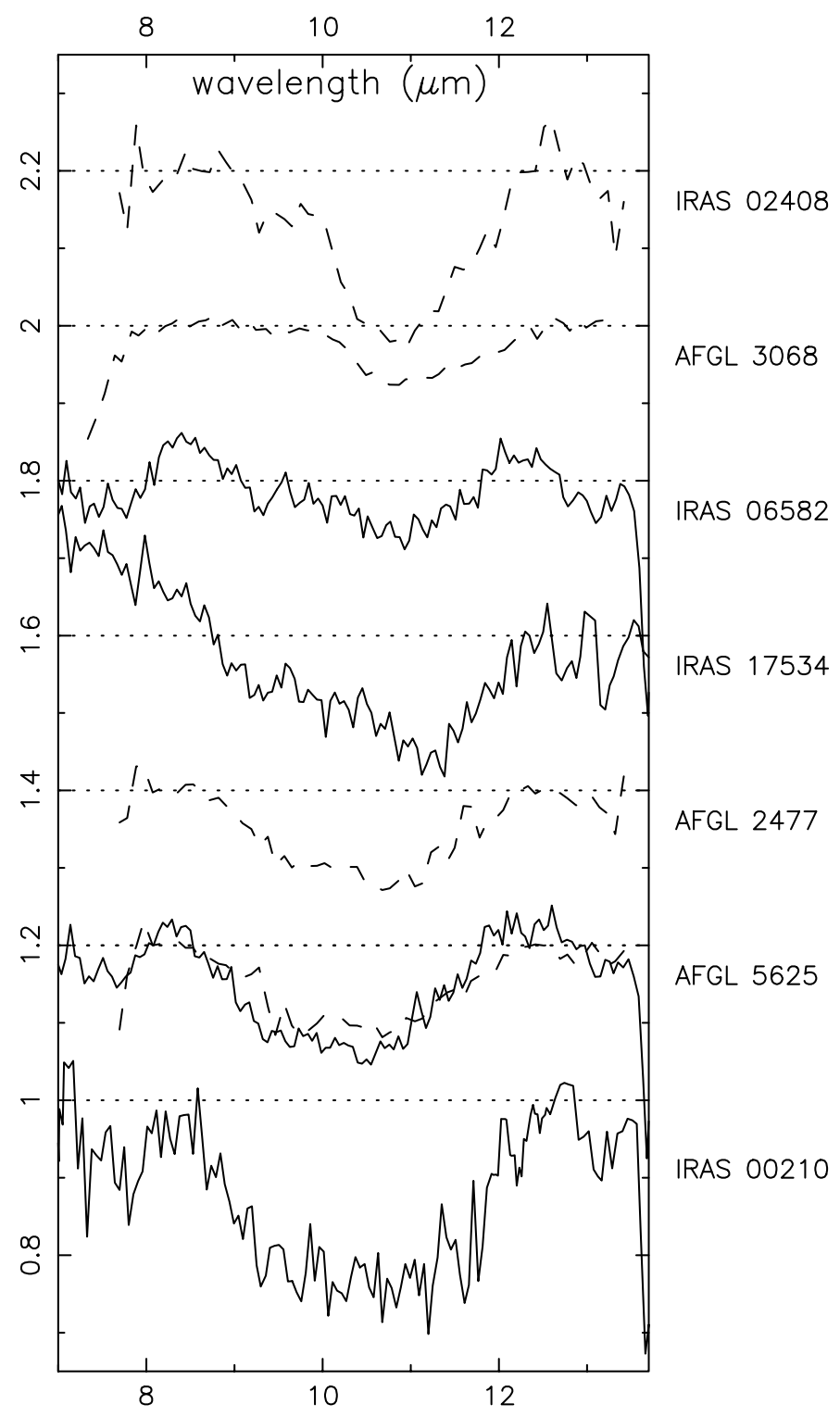

FIG. 5.-Continuum-divided 7-13 $\mu \mathrm{m}$ spectra of extreme carbon stars. Solid lines: ISO SWS spectra divided by model fits from Volk et al. (2000). Dashed lines: UKIRT CGS3 spectra divided by best-fit blackbody dust continuum from Speck et al. (1997). Spectra are offset in the $y$-direction for clarity: IRAS 00210+ 6221 is not offset; AFGL 5625 is offset by +0.2 ; AFGL 2477 is offset by 0.4 ; IRAS $17534-3030$ is offset by 0.6 ; IRAS $06582+1507$ is offset by 0.8 ; AFGL 3068 is offset by 1.0 ; and IRAS $02408+5458$ is offset by 1.2 .

emergence of a second spectral feature at $\sim 9 \mu \mathrm{m}$. Sloan et al. (1998) found an anticorrelation between the $\sim 9 \mu \mathrm{m}$ feature and the decreasing continuum temperature, which is opposite to what was observed by both Baron et al. (1987) and Goebel et al. (1995). With such a diverse set of analyses and interpretations of what is essentially the same data set (except for Speck et al. 1997), no clear correlation can be discerned between changes in the spectral features and evolution of the dust. The differences in interpretation of the IRAS LRS data may stem from differing underestimations of the depth of the molecular ( $\mathrm{HCN}$ and $\mathrm{C}_{2} \mathrm{H}_{2}$ ) absorptions and thus the continuum level (Aoki et al. 1999).

\subsection{The $\mathrm{SiC}$ Absorption Feature}

The prototype for extreme carbon stars is AFGL 3068, which has an absorption feature at $\sim 11 \mu \mathrm{m}$, tentatively attributed to 


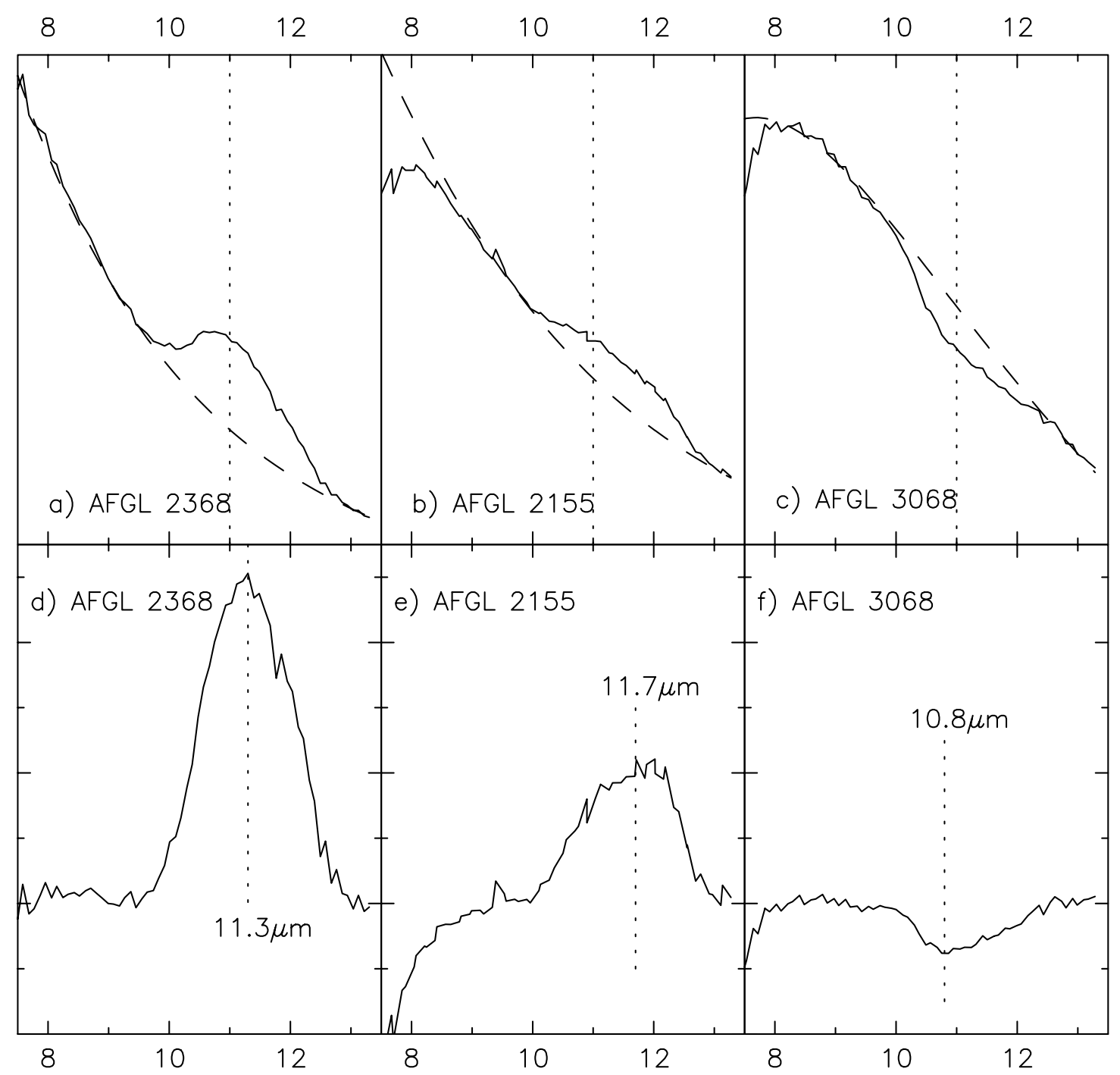

FIG. 6. - UKIRT CGS3 mid-infrared spectra of carbon stars from Speck et al. (1997). Top: Solid line, Spectrum $\left(F_{\lambda}\right)$; dashed line, continuum. Bottom: Continuum divided spectra. $(a, d)$ Pure $\mathrm{SiC}$ emission. $(b, e)$ Self-absorbed $\mathrm{SiC}$ emission. $(c, f) \mathrm{SiC}$ (self-)absorption. The $x$-axis is wavelength in $\mu \mathrm{m}$.

absorption by SiC (Jones et al. 1978). Speck et al. (1997) reinvestigated AFGL 3068 and confirmed the absorption features. In addition, Speck et al. (1997) discovered three more extreme carbon stars (IRAS 02408+5458, AFGL 2477, and AFGL 5625) with $\sim 11 \mu \mathrm{m}$ absorption features attributable to SiC. Two of these objects (AFGL 2477 and AFGL 5625) exhibited a doublepeaked absorption feature, with the $\sim 11 \mu$ m feature accompanied by a shorter wavelength absorption peak at $\sim 9 \mu \mathrm{m}$. Speck et al. (1997) attributed this shorter wavelength peak to interstellar silicate absorption along the line of sight, although it is possible that this feature is related to the $\sim 9 \mu \mathrm{m}$ emission feature that accompanies the $\sim 11 \mu \mathrm{m}$ emission feature in the spectra of some carbon stars. In all four cases, the $\sim 11 \mu$ m absorption feature actually "peaks" at $10.8 \mu \mathrm{m}$.

The absorption features of AFGL 3068 and IRAS 02408+ 5458 were revisited by Clément et al. (2003), who showed that these features, as seen in Infrared Space Observatory (ISO) Short Wavelength Spectrometer (SWS) spectra, were consistent with isolated $\beta$-SiC nanoparticles. However, they could not fit the short-wavelength side of the $\sim 11 \mu \mathrm{m}$ absorption feature using $\mathrm{SiC}$ alone, which may indicate that the $\sim 9 \mu \mathrm{m}$ feature absorption is intrinsic to these stars and that its strength varies. This is discussed further in $\S 2.3$.
The double-peaked absorption features of AFGL 2477 and AFGL 5625 were revisited by Clément et al. (2005). This work presented new infrared spectra of silicon nitride $\left(\mathrm{Si}_{3} \mathrm{~N}_{4}\right)$ and found a correlation between the observed double-peaked feature and the laboratory absorption spectrum. Furthermore, they were able to correlate various weaker longer wavelength absorptions in the astronomical spectra with those of $\mathrm{Si}_{3} \mathrm{~N}_{4}$ observed in the laboratory. However, while the match to the longer wavelength features is good, in their laboratory spectra the relative strength of the $\sim 11$ and $\sim 9 \mu \mathrm{m}$ features compared to the longer wavelength features indicates that $\mathrm{Si}_{3} \mathrm{~N}_{4}$ is almost certainly present but cannot be solely responsible for these $\sim 11$ and $\sim 9 \mu$ m absorption features.

Volk et al. (2000) presented the ISO spectra of five extreme carbon stars. They produced radiative transfer models, assuming amorphous carbon dust and including a way to fit the broad 26-30 $\mu \mathrm{m}$ feature, but without trying to fit the absorption features in the $8-13 \mu \mathrm{m}$ range. We have divided the $I S O$ spectra by their model fits, and the resulting spectrum of the 7-13 $\mu \mathrm{m}$ region is shown in Figure 5, together with the continuumdivided spectra of extreme carbon stars from Speck et al. (1997). It is clear that IRAS $06582+1507$ shows the $\sim 11 \mu \mathrm{m}$ absorption feature seen in AFGL 3068 and IRAS 02408+5458. The 
spectrum of IRAS $00210+6221$ shows a double/broad feature similar to that of AFGL 5625. IRAS 17534-3030 seems to be intermediate between the two. Given that the extreme carbon star phase is not expected to last more than a few $\times 10^{4} \mathrm{yr}$, it is not surprising that stars that exhibit these features are rare. We now have four stars that exhibit the single $\sim 11 \mu \mathrm{m}$ absorption feature and three that exhibit the broader double feature. It is no longer possible to ignore these absorption features when trying to understand the evolution of dust around carbon stars.

The $\sim 9 \mu \mathrm{m}$ feature/wing appears to correlate with optical depth, appearing strongest when the $\sim 11 \mu \mathrm{m}$ feature is in absorption, but it also exists when the $\sim 11 \mu \mathrm{m}$ feature region weakly emits. This feature may be due to amorphous $\mathrm{SiC}$ with excess carbon (see $\S 2$ ), in which case the formation of such grains occurs when the dust shell is denser, further up the AGB. This makes sense, in that the high-density shells may form dust grains so fast that the atoms do not have time to migrate to the most energetically favored position before another atom sticks on. In this way, we would expect to form amorphous rather than crystalline grains. If the double-peaked absorption feature is due to nanocrystalline grains, this further supports the hypothesis that grain sizes decrease as mass-loss rates increase. Furthermore, early in the life of a carbon star the dust-forming regions will have more $\mathrm{Si}$ than $\mathrm{C}$ for dust formation (the majority of the $\mathrm{C}$ atoms will be locked into $\mathrm{CO}$ molecules). As the star evolves and more carbon is dredged up from deep within the star, there will eventually be more $\mathrm{C}$ atoms than $\mathrm{Si}$ atoms, and excess $\mathrm{C}$ will get trapped in the grains.

The lack of amorphous $\mathrm{SiC}$ in meteoritic samples may be due to the relative scarcity of this form of $\mathrm{SiC}$ compared to the crystalline polytypes. A typical AGB star sends dust out into the interstellar medium for a few hundred thousand years, but the extreme carbon star phase is much shorter lived $\left(<10^{4} \mathrm{yr}\right)$ and may not occur for all carbon stars (absorption features are rare). Therefore, we would expect there to be much more crystalline (mostly $\beta$-SiC) than amorphous $\mathrm{SiC}$ grains. Furthermore, there may well be a mixture of amorphous and $\beta$-SiC forming in the circumstellar shells of very evolved (extreme) AGB stars. Alternatively, the meteoritic data may support the attribution of the 9 and $21 \mu \mathrm{m}$ features to nanocrystalline $\mathrm{SiC}$ grains with diamond inclusions. Nanometer-sized SiC grains have been found in the presolar SiC samples (Bernatowicz et al. 2005).

\subsection{Carbon-rich Post-AGB Spectra: The $21 \mu \mathrm{m}$ Feature}

Among the C-rich PPNe, approximately half exhibit a feature in their infrared spectra at $21 \mu \mathrm{m}$ (Omont et al. 1995). Subsequent higher resolution data revised the so-called $21 \mu \mathrm{m}$ position to $20.1 \mu \mathrm{m}$ (Volk et al. 1999). PPNe that display this feature are all C-rich and all show evidence of $s$-process enhancements in their photospheres, indicative of efficient dredge-up during the ascent of the AGB (Van Winckel \& Reyniers 2000). The $21 \mu \mathrm{m}$ feature is rarely seen in the spectra of either the PPN precursors, AGB stars, or their successors, PNe (however, see Volk et al. 2000; Hony et al. 2003). The observed peak positions and profile shapes of the $21 \mu \mathrm{m}$ feature are remarkably constant (Volk et al. 1999). This enigmatic feature has been widely discussed since its discovery (Kwok et al. 1989) and has been attributed to a variety of both transient molecular and long-lived solid-state species, but most of these species have since been discarded as carriers, except for hydrogenated amorphous carbons (HACs)/polycyclic aromatic hydrocarbons (PAHs; Justtanont et al. 1996; Volk et al. 1999; Buss et al. 1990; Grishko et al. 2001), and $\mathrm{SiC}$ (Speck \& Hofmeister 2004). In the case of SiC, it is necessary for the dust grains to be small and contaminated with carbon impurities in order for this feature to appear (Kimura et al. 2005a, 2005b). Furthermore, the cooling and thinning of the dust shell is essential to the emergence of this feature. During the AGB phase, the dust is too warm for this feature to appear, but as the dust cools the $\sim 11 \mu \mathrm{m}$ feature of $\mathrm{SiC}$ is diminished and the $21 \mu \mathrm{m}$ feature is promoted by the underlying dust continuum emission. In this case, the changing spectrum reflects the change in temperature and optical depth of the dust shell, whereas the spectral features are indicative of the last stage of carbonrich evolution (the extreme carbon star phase).

\section{SELF-ABSORPTION: THE EFFECT OF CHANGING GRAIN SIZES}

Cohen (1984) interpreted the change in the appearance of $\sim 11 \mu \mathrm{m}$ feature from sharp and narrow to broad and flat topped as possibly an effect of self-absorption. This was supported by Speck et al. (1997), who showed that all carbon star spectra in their sample with dust continuum temperatures less than $1200 \mathrm{~K}$ needed to have self-absorbed $\mathrm{SiC}$ in order to be fitted well. Selfabsorption exhibits some interesting characteristics that can be used to diagnose the nature of the dust grains that produce observed spectra.

Figure 7 shows how the absorption profile changes as particle thickness (i.e., grain size) increases. This appears to be a violation of Beer's law, in that the absorbance is not simply increasing (for a single wavelength) with thickness of the particles. This departure occurs because the measured intensity depends not only on the amount of light that the sample absorbs but also on the amount reflected at the surface facing the source. The main peak (the TO mode) becomes saturated in measured spectra when the actual amount of light transmitted at the TO frequency equals the reflectivity. This point is reached at lower thicknesses (grain sizes) for the strong TO mode than for the more weakly absorbing shoulder. Violations of Beer's law will alter self-absorption in astronomical environments but not emission spectra, because the latter are not affected by surface reflections. Therefore, emission spectra of the grains are not so sensitive to the grain size. As long as the grains are still small compared to the wavelength at which they are observed (i.e., grains smaller than $\approx 1 \mu \mathrm{m}$ for $10 \mu \mathrm{m}$ spectra), the pure emission feature will appear at the same wavelength $(\sim 11.3 \mu \mathrm{m})$. However, once the grains are cool enough and the optical depth is high enough, self-absorption of the $11.3 \mu \mathrm{m}$ feature will begin and the profile of the feature will depend on the relative sizes of the grains.

In computing the effect of Beer's law violations on selfabsorption, we assume that the following hold: (1) the absorbing particles are thicker (larger) than the emitting particles; (2) the light received by the absorbing particles is that of the star, $I_{0}$, i.e., the inner cloud of emitting particles is rarefied enough that it contributes negligible intensity compared to the star; and (3) the outer dust transmits more light that the inner dust emits (i.e., starlight is also transmitted).

Beer's law states that the wavelength-dependent absorbance $a(\lambda)$ of a given substance is proportional to the mass absorption coefficient $\kappa_{\text {abs }}(\lambda)$ and the thickness through which the light has to pass $d$,

$$
a(\lambda)=\kappa_{\mathrm{abs}}(\lambda) d
$$

Deviations from Beer's law may occur for many reasons. Strictly, Beer's law applies to transparent particles (i.e., particles that transmit some light at all wavelengths), and therefore at high concentrations/opacities may no longer be applicable. Other problems include stray light (McMillan \& Hofmeister 1988). 


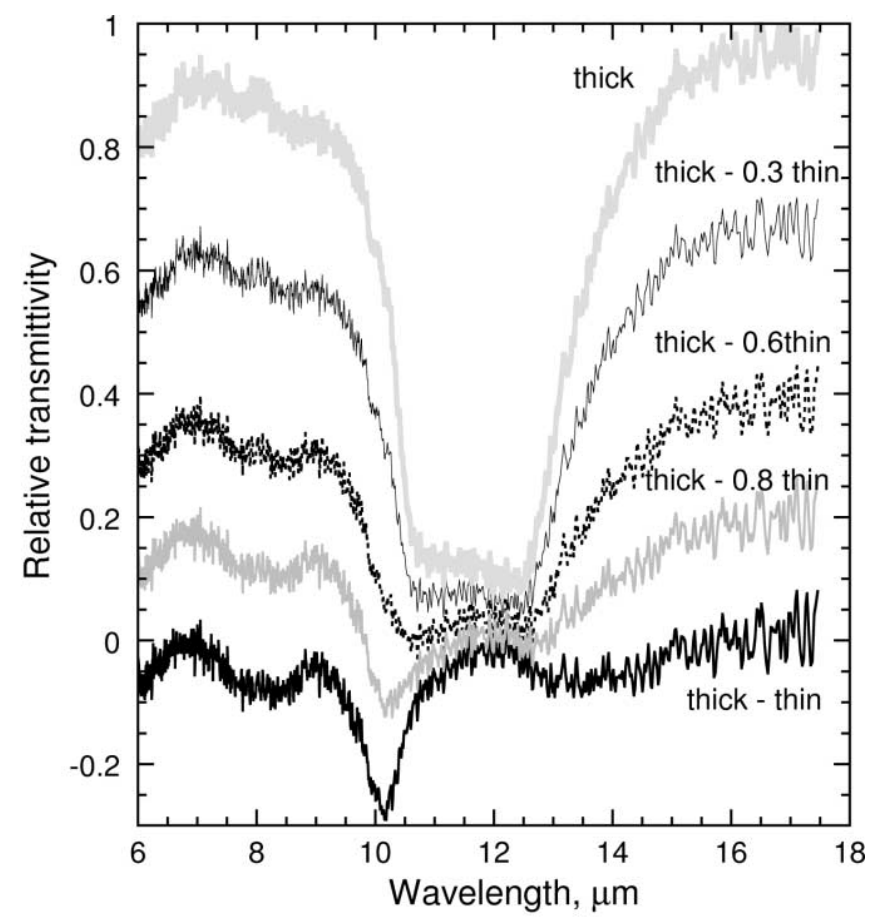

FIG. 7.-Effect of thickening dust samples. The fractional factor is $f$ (see eq. [1]), which accounts for more or less light coming from the emitting grains. If the grains are all the same size, the absorption feature is in the same position as the emission feature. If the absorbing grains are larger, then there is an apparent shift in the feature position.

When measuring absorption, we usually measure the transmittance $T(\lambda)$ of a material and thus obtain the absorptivity ${ }^{3}$ $A(\lambda)$ as

$$
\begin{gathered}
T=\frac{I_{\text {trans }}}{I_{0}}=1-\frac{I_{\mathrm{abs}}}{I_{0}}=1-e^{-a}, \\
A=\frac{I_{\mathrm{abs}}}{I_{0}}=e^{-a} .
\end{gathered}
$$

The light received from a circumstellar shell $I_{\text {dust }}$ is given by

$$
I_{\text {dust }}=I_{\text {emit }}+I_{\text {trans }} \text {, }
$$

where $I_{\text {emit }}$ is the emission from the inner dust and $I_{\text {trans }}$ is the transmission from the outer dust.

From Kirchhoff's law, the emissivity is the same as the absorptivity, so that $I_{\text {emit }}=I_{\text {abs }}$. Therefore,

$$
\begin{aligned}
\frac{I_{\text {dust }}}{I_{0}} & =f \frac{I_{\mathrm{abs}}}{I_{0}}+\frac{I_{\text {trans }}}{I_{0}}, \\
\frac{I_{\text {dust }}}{I_{0}} & =f e^{-a}+1-e^{-a},
\end{aligned}
$$

where the factor $f$ varies from 0 to 1 as the fraction of light originating from emitting dust increases.

The absorption coefficient will change with grain size $d$ because of the effect of reflections,

$$
\frac{I_{\text {dust }}}{I_{0}}=f e^{-\kappa_{\text {abs, inner }} d_{\text {inner }}}+1-e^{-\kappa_{\text {abs,outer }} d_{\text {outer }}} .
$$

\footnotetext{
${ }^{3}$ Note that absorbance is the reciprocal of the log of the transmittance, in contrast to the absorptivity defined here.
}

Essentially, equation (1) provides the effective transmission of the dust. If Beer's law is followed (i.e., reflectivity is low and the particles are thin enough to transmit light at all frequencies), then $\kappa_{\text {abs,inner }}=\kappa_{\text {abs, outer }}$ and the spectrum received is no different from the intrinsic absorptions. However, if the absorbing particles are larger and opaque at some frequencies (i.e., the TO mode), the spectrum will be altered. We have computed the effect of particle thickness on self-absorption in circumstellar shells, and the resulting spectra are shown in Figure 7. For $\kappa_{\text {abs, outer }} d_{\text {outer }}$ we used baseline-corrected absorption spectra from the thickest sample of nano- $\beta$-SiC shown in Figure 2, and for $\kappa_{\text {abs, inner }} d_{\text {inner }}$ we similarly used the intermediate sample. The same results would be obtained for the thinnest sample. As the contribution of the emitting particles increases, the contribution of the LO component increases relative to the TO component, and the TO component appears to shift to longer wavelengths.

If the size and absorption coefficients are identical for both the inner and outer regions of the dust shell, then there will be no shift in the spectral features between emission and absorption. However, if the outer grains are larger, there will be a shift in the absorption to shorter wavelengths, as shown in Figure 7.

In terms of what we would expect to see in the sequence of carbon star spectra, the discussion above means that for optically thin dust shells, where we are seeing pure emission, the $\mathrm{SiC}$ feature should peak at $11.3 \mu \mathrm{m}$ and be sharp. As the optical depth goes up, the $\mathrm{SiC}$ feature will become self-absorbed, but if the grains in the outer part of the shell are larger than those in the inner zone, the absorption will occur preferentially on the LO side of the feature, diminishing the blue side, which would appear as a shift in the feature to longer wavelengths $(\sim 11.7 \mu \mathrm{m})$. As the optical depth gets high enough for the emission feature to be completely absorbed, we no longer see the $11.7 \mu \mathrm{m}$ feature, and the absorption will peak at a shorter wavelength $(10.8 \mu \mathrm{m})$ than the regular $\mathrm{SiC}$ feature at $11.3 \mu \mathrm{m}$. This is shown schematically in Figure 8 (cf. Fig. 6). This mechanism explains the evolution in the observed $\mathrm{SiC}$ absorption feature in extreme carbon stars. Furthermore, it correlates with the evolution of the

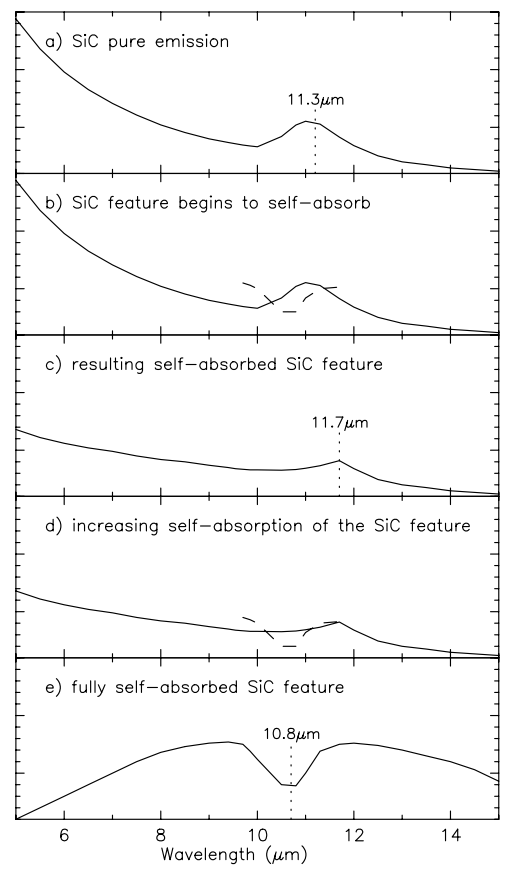

FIG. 8.-Schematic of self-absorption and its effect on the peak position of the $\sim 11 \mu \mathrm{m}$ feature. 
spectral features as seen by Baron et al. (1987), who showed that as the continuum temperature decreases and the peak continuum strength of the $\mathrm{SiC}$ feature diminishes, the peak position tends to move from $\sim 11.3$ to $\sim 11.7 \mu \mathrm{m}$. This suggests that there is an evolution in the dust grains toward smaller dust grains with higher mass loss.

Two separate studies of presolar $\mathrm{SiC}$ suggest that there is an evolution in grain size that corresponds to the evolution of carbon stars. Prombo et al. (1993) found a correlation between grain size and the concentration of $s$-process elements in $\mathrm{SiC}$ grains taken from the Murchison meteorites. The Indarch meteorite presolar SiC grains yielded similar results (Jennings et al. 2002). In both cases, the smaller grains have higher relative abundances of $s$-process elements. Since newly formed $s$-process elements are dredged up along with carbon from the He-burning shell, they are more abundant in more evolved circumstellar shells than in early ones. Therefore, these results suggest that the grains formed around carbon stars decrease in size as the stars evolve and support the observations of the self-absorption effects described above.

Up until now, the generally accepted wisdom has been that low mass-loss rates early in the AGB phase lead to small grains, and increasing mass-loss rates permit the growth of larger grains. In the new scenario, smaller grain sizes with increasing mass loss can be understood in terms of potential nucleation sites. The hardest step in grain formation is the production of seeds onto which minerals can grow. In a low-density gas very few seeds can nucleate, leading to the formation of few grains. However, these few grains can grow large, because there are not so many grains competing for the same atoms. Conversely, in a highdensity gas it is easier to form seeds, but so many more nucleate that there are not enough atoms around for any individual grain to grow large. In this way, high densities lead to very dense dust shells of small grains. This effect may be exacerbated by the earlier outflows moving slower than the later outflows. If the stellar winds that drive the mass loss and send the newly formed grains away from the star accelerate with time, then the earlier grains will have more time closer to the star in regions dense enough for grain growth to occur. Since radiation-pressure coupling should be related to grain composition, and this will vary with the $\mathrm{Si} / \mathrm{C}$ ratio, we may expect such an effect.

\section{CONCLUSIONS}

The dust shells around carbon stars and their successors, carbon-rich post-AGB stars, evolve along with the star. In early phases, the mass-loss rate is low, and hence the dust shell is optically thin. As these stars evolve, the mass-loss rate increases, and thus the dust shell gets more optically thick, until the star is completely obscured in visible light and very bright in the infrared. During this evolution the relative abundance of silicon to carbon $(\mathrm{Si} / \mathrm{C})$ available for dust formation decreases, so that in the early carbon stars there is more $\mathrm{Si}$ and $\mathrm{C}$, whereas in the extreme carbon stars there is much more $\mathrm{C}$ than $\mathrm{Si}$. Once the AGB phase ends, the existing dust shell spreads out, becoming optically thin and cooler. The combination of increasing density and increasing carbon on the AGB manifests itself in the nature of the dust grains, as seen in the spectral sequence for carbon stars.

Early in the carbon star phase, when the mass-loss rate is low and the shell is optically thin, the $\sim 11 \mu \mathrm{m} \mathrm{SiC} \mathrm{emission}$ feature is strong, narrow, and sharp. As the mass loss increases and the shell becomes optically thicker, the SiC feature broadens, flattens, and weakens. Finally, once the mass-loss rate is extremely high and the shell is extremely optically thick, the $\mathrm{SiC}$ feature appears in absorption. The shifts in the peak of the $\sim 11 \mu \mathrm{m} \mathrm{SiC}$ feature are attributable to a combination of optical depth and grain sizes. The only way to see a shift in the absorption feature to $10.8 \mu \mathrm{m}$ is if the absorbing grains are larger than the emitting grains. Therefore, we have observational evidence to suggest that the grains formed in the circumstellar shell get smaller as the star evolves. Further evidence for this scenario is seen both in post-AGB spectra and in meteoritic studies of presolar grains.

We have presented new mid-IR laboratory spectra for various forms of $\mathrm{SiC}$ including amorphous $\mathrm{SiC}$ with excess carbon structure. These data corroborate and augment the laboratory work of Kimura \& Kaito (2003) and Kimura et al. (2005a, 2005b), indicating that solid solutions of $\mathrm{C}$ replacing $\mathrm{Si}$ in $\mathrm{SiC}$ have the diamond structure on a local scale. This is a good candidate for the carrier of the $\sim 9 \mu \mathrm{m}$ feature seen in both emission and absorption and correlated with trends in the $\sim 11 \mu \mathrm{m}$ feature.

We are extremely grateful to the reviewer, whose comments significantly improved the paper. We would also like to thank Kevin Volk for providing us with his ISO SWS spectra and model fits for extreme carbon stars. Support for A. M. H. was provided by NASA grant APRA04-0000-0041.

\section{REFERENCES}

Aoki, W., Tsuji, T., \& Ohnaka, K. 1999, A\&A, 350, 945

Axelbaum, R. L., Lottes, C. R., Rosen, L. J., \& Huertas, J. I. 1996, in 26th International Symposium on Combustion (Pittsburgh: Combustion Institute), 1891

Baron, Y., de Muizon, M., Papoular, R., \& Pégourié, B. 1987, A\&A, 186, 271

Bernatowicz, T., Fraundorf, G., Tang, M., Anders, E., Wopenka, B., Zinner, E., \& Fraundorf, P. 1987, Nature, 330, 728

Bernatowicz, T. J., et al. 2005, in Meteorites and the Early Solar System II, ed. D. Lauretta \& H. Y. McSween, Jr. (Tucson: Univ. Arizona Press), in press

Buss, R. H., Jr., Cohen, M., Tielens, A. G. G. M., Werner, M. W., Bregman, J. D., Witteborn, F. C., Rank, D., \& Sandford, S. A. 1990, ApJ, 365, L23

Chan, S. J., \& Kwok, S. 1990, A\&A, 237, 354

Clément, D., Mutschke, H., Klein, R., \& Henning, T. 2003, ApJ, 594, 642

Clément, D., Mutschke, H., Klein, R., Jäger, C., Dorschner, J., Sturm, E., \& Henning, Th. 2005, ApJ, 621, 985

Cohen, M. 1984, MNRAS, 206, 137

Daulton, T. L., Bernatowicz, T. J., Lewis, R. S., Messenger, S., Stadermann, F. J., \& Amari, S. 2003, Geochim. Cosmochim. Acta, 67, 4743

Friedemann, C. 1969, Physica, 41, 189

Gilman, R. C. 1969, ApJ, 155, L185
Gilra, D. P. 1971, Nature, 229, 237

1972, in Scientific Results from the Orbiting Astronomical Observatory $(O A O-2)$, ed. A. D. Code (NASA SP-310; Washington: NASA), 295

Goebel, J. H., Cheeseman, P., \& Gerbault, F. 1995, ApJ, 449, 246

Grishko, V. I., Tereszchuk, K., Duley, W. W., \& Bernath, P. 2001, ApJ, 558, L129

Hackwell, J. A. 1972, A\&A, 21, 239

Hofmeister, A. M., Keppel, E., \& Speck, A. K. 2003, MNRAS, 345, 16

Hofmeister, A. M., Rosen, L., \& Speck, A. K. 2000, in ASP Conf. Ser. 196, Thermal Emissions Spectroscopy and Analysis of Dust, Disks and Regoliths, ed. M. Sitko, A. L. Sprague, \& D. K. Lynch (San Francisco: ASP), 291

Hony, S., Tielens, A. G. G. M., Waters, L. B. F. M., \& de Koter, A. 2003, A\&A, 402, 211

Jennings, C. L., Savina, M. R., Messenger, S., Amari, S., Nichols, R. H., Jr., Pellin, M. J., \& Podosek, F. A. 2002, Lunar Planet. Sci. Conf., 33, 1833

Jones, B., Merrill, K. M., Puetter, R. C., \& Willner, S. P. 1978, AJ, 83, 1437

Justtanont, K., Barlow, M. J., Skinner, C. J., Roche, P. F., Aitken, D. K., \& Smith, C. H. 1996, A\&A, 309, 612

Kimura, Y., \& Kaito, C. 2003, J. Crystal Growth, 255, 282

Kimura, Y., Sato, T., \& Kaito, C. 2005a, Carbon, 43, 1570

Kimura, Y., et al. 2005b, J. Crystal Growth, 275, 977 
Kwok, S., Volk, K., \& Hrivnak, B. J. 1989, ApJ, 345, L51

Little-Marenin, I. R. 1986, ApJ, 307, L15

McMillan, P. F., \& Hofmeister, A. M. 1988, in Spectroscopic Methods in Mineralogy and Geology, ed. F. C. Hawthorne (Washington: Mineralogical Society of America), 99

Omont, A., et al. 1995, ApJ, 454, 819

Prombo, C. A., Podosek, F. A., Amari, S., \& Lewis, R. S. 1993, ApJ, 410, 393

Sloan, G. C., Little-Marenin, I. R., \& Price, S. D. 1998, AJ, 115, 809

Speck, A. K., Barlow, M. J., \& Skinner, C. J. 1997, MNRAS, 288, 431
Speck, A. K., \& Hofmeister, A. M. 2004, ApJ, 600, 986 Speck, A. K., Hofmeister, A. M., \& Barlow, M. J. 1999, ApJ, 513, L87 Spitzer, W. G., Kleinmann, D. A., \& Walsh, D. 1959, Phys. Rev., 113, 127 Treffers, R., \& Cohen, M. 1974, ApJ, 188, 545

Van Winckel, H., \& Reyniers, M. 2000, A\&A, 354, 135

Volk, K., Kwok, S., \& Hrivnak, B. J. 1999, ApJ, 516, L99

Volk, K., Kwok, S., \& Langill, P. P. 1992, ApJ, 391, 285

Volk, K., Xiong, G.-Z., \& Kwok, S. 2000, ApJ, 530, 408

Willems, F. J. 1988, A\&A, 203, 51 